\title{
Expanding the knowledge about the occurrence of anurans in the highest amphibian diversity area of Atlantic Forest: Parque Estadual da Serra do Mar, São Paulo, Brazil
}

\author{
Fernando Rodrigues da Silva', Mariana Lúcio Lyra' ${ }^{2}$, Célio Fernando Baptista Haddad ${ }^{2}$ \& \\ Denise de Cerqueira Rossa-Feres ${ }^{3}$ \\ ${ }^{1}$ Universidade Federal de São Carlos, Departamento de Ciências Ambientais, Laboratório de Ecologia Teórica: \\ Integrando Tempo, Biologia e Espaço (LET.IT.BE), Sorocaba, São Paulo, Brazil \\ ${ }^{2}$ Universidade Estadual Paulista, Departamento de Zoologia e Centro de Aquicultura da UNESP (CAUNESP), \\ Instituto de Biociências, Rio Claro, SP, Brazil \\ ${ }^{3}$ Universidade Estadual Paulista, Departamento de Zoologia e Botânica, São José do Rio Preto, São Paulo, Brazil \\ *Corresponding Author: Dr. Fernando Rodrigues da Silva, e-mail: fernando.ecologia@gmail.com
}

DA SILVA, F.R., LYRA, M.L., HADDAD, C.F.B., ROSSA-FERES, D.C. Expanding the knowledge about the occurrence of anurans in the highest amphibian diversity area of Atlantic Forest: Parque Estadual da Serra do Mar, São Paulo, Brazil. Biota Neotropica. 17(2): e20160282. http://dx.doi.org/10.1590/1676-0611-BN-2016-0282

\begin{abstract}
The vegetation cover in the Atlantic Forest has been converted to human use or degraded by human activities, which declined the vegetation to $16 \%$ of its original extent. Although several protected areas have been created in this Neotropical biome over the past decades, our knowledge of the amphibian species within these areas is still far from adequate. Here, we present lists of anuran species from three different areas of Parque Estadual da Serra do Mar: núcleos Curucutu, Santa Virgínia and São Sebastião. To survey anuran species, we visited six sampling units (two ponds, two streams and two trails inside forest fragments) in each locality. Samplings were accomplished from December 2014 to February 2015, December 2015 to February 2016 and December 2016 to February 2017, totalizing 27 days of field samplings in each sampling unit. We recorded 34 anuran species in the Núcleo Curucutu, 44 species in the Núcleo São Sebastião and 42 species in the Núcleo Santa Virgínia, totalizing 65 species belonging to 12 families (number of species in parentheses): Bufonidae (5), Brachycephalidae (6), Centrolenidae (1), Craugastoridae (1), Cycloramphidae (2), Hemiphractidae (1), Hylidae (29), Hylodidae (3), Leptodactylidae (12), Microhylidae (2), Odontophrynidae (2), and Phyllomedusidae (1). We hope that these lists can be useful for future studies as well as helping in the management and conservation planning of these protected areas.
\end{abstract}

Keywords: Amphibians, Biodiversity, Conservation, Hotspots, Species list.

\section{Expandindo o conhecimento sobre a ocorrência de anuros na área com maior diversidade de anfíbios da Floresta Atlântica: Parque Estadual da Serra do Mar, São Paulo, Brasil}

\begin{abstract}
Resumo: A cobertura vegetal da Floresta Atlântica foi convertida para uso humano ou degradada pela atividade humana permanecendo atualmente $16 \%$ da sua extensão original. Embora muitas áreas protegidas tenham sido criadas neste bioma neotropical nas últimas décadas, nosso conhecimento sobre as espécies de anfíbios nestas áreas ainda está longe do adequado. Aqui, nós apresentamos listas das espécies de anuros para três diferentes áreas do Parque Estadual da Serra do Mar: núcleos Curucutu, Santa Virgínia e São Sebastião. Para amostrar as espécies de anuros, visitamos seis locais de amostragem (duas poças, dois riachos e duas trilhas) em cada localidade. As amostragens foram realizadas de dezembro 2014 a fevereiro de 2015, dezembro de 2015 a fevereiro de 2016 e de dezembro de 2016 a fevereiro de 2017, totalizando 27 dias de amostragens de campo em cada ponto de amostragem. Registramos 34 espécies de anuros no Núcleo Curucutu, 44 espécies no Núcleo São Sebastião, e 42 espécies no Núcleo Santa Virgínia, totalizando 65 espécies pertecentes a 12 famílias (número de espécies entre parênteses): Bufonidae (5), Brachycephalidae (6), Centrolenidae (1), Craugastoridae (1), Cycloramphidae (2), Hemiphractidae (1), Hylidae (29), Hylodidae (3), Leptodactylidae (12), Microhylidae (2), Odontophrynidae (2), e Phyllomedusidae (1). Esperamos que estas listas possam ser úteis para futuros estudos assim como auxiliar os planos de manejo e conservação destas áreas protegidas.
\end{abstract}

Palavras chaves: Anfíbios, Biodiversidade, Conservação, Hotspots, Lista de espécies. 


\section{Introduction}

The Atlantic Forest, a global hotspot for biodiversity conservation (Mittermeier et al. 2004), originally covered 1.3 million $\mathrm{km}^{2}$, of which between $84 \%$ and $89 \%$ have been converted to human use or degraded by human activities (Ribeiro et al. 2009). This biome harbors one of the highest diversity of amphibians in the world, hosting approximately 600 species of amphibians (Haddad et al. 2013). Although several protected areas have been created in this Neotropical biome over the past three decades, our knowledge of the amphibian species within these areas is still far from adequate. For example, the Parque Estadual da Serra do Mar (PESM) in the state of São Paulo, Brazil, is the largest protected area of the entire Atlantic Forest biome but some administrative areas such as Núcleo São Sebastião still do not have an amphibian survey published. This is worrisome because PESM harbors the highest amphibian diversity of the Atlantic Forest biome (Loyola et al. 2014). Here, we surveyed amphibian species of Núcleo Curucutu, Núcleo Santa Virgínia and Núcleo São Sebastião and present the first list of amphibian species for Núcleo São Sebastião. Natural history knowledge and species composition are the basic dataset for ecology, systematics, biogeography, and conservation biology (e.g., Collen et al. 2008, Da Silva et al. 2012, 2014); therefore, we hope that our lists can be useful for future studies as well as helping in the management and conservation planning of these protected areas.

\section{Material and Methods}

\section{Study area}

The PESM $\left(23^{\circ} 13\right.$ '8.4'S and $24^{\circ} 5^{\prime} 42.0^{\prime \prime} \mathrm{S}, 44^{\circ} 43^{\prime} 12.0^{\prime} \mathrm{W}$ and $46^{\circ} 33^{\prime} 50.4^{\prime \prime} \mathrm{W}$ ) is the largest protected area in the Atlantic Forest biome with 315,390 ha in the state of São Paulo, southeastern Brazil (Figure 1). Due to its large extension the PESM is managed by 11 administrative divisions named: Núcleo Bertioga, Núcleo Caminhos do Mar, Núcleo Caraguatatuba, Núcleo Cunha, Núcleo Curucutu, Núcleo Itariru, Núcleo Itutinga Pilões, Núcleo Padre Dória, Núcleo Picinguaba, Núcleo Santa Virgínia and Núcleo São Sebastião. In this study, we carried out species inventories in three of these localities (Figure 1): (i) Núcleo Curucutu (23 $59^{\prime} 6.0^{\prime \prime} \mathrm{S}$ and $46^{\circ} 44^{\prime} 34.8^{\prime \prime} \mathrm{W}$ ), with 12,029 ha is encompassed by four municipalities (Itanhaém, Juquitiba, Mongaguá and São Paulo) and altitudes varying from 15 to $870 \mathrm{~m}$ asl; (ii) Núcleo Santa Virgínia (2321'3.6”S and $45^{\circ} 8^{\prime} 6.0^{\prime \prime} \mathrm{W}$ ), with 17,500 ha is encompassed by five municipalities (São Luiz do Paraitinga, Cunha, Caraguatatuba, Ubatuba and Natividade da Serra), and altitudes varying from 740 to $1620 \mathrm{~m}$ asl; and (iii) Núcleo São Sebastião (2343'1.2”S and 4542'10.8”W), with 26,268 ha is encompassed by the municipality of São Sebastião and altitudes varying from sea level to $1200 \mathrm{~m}$ asl.

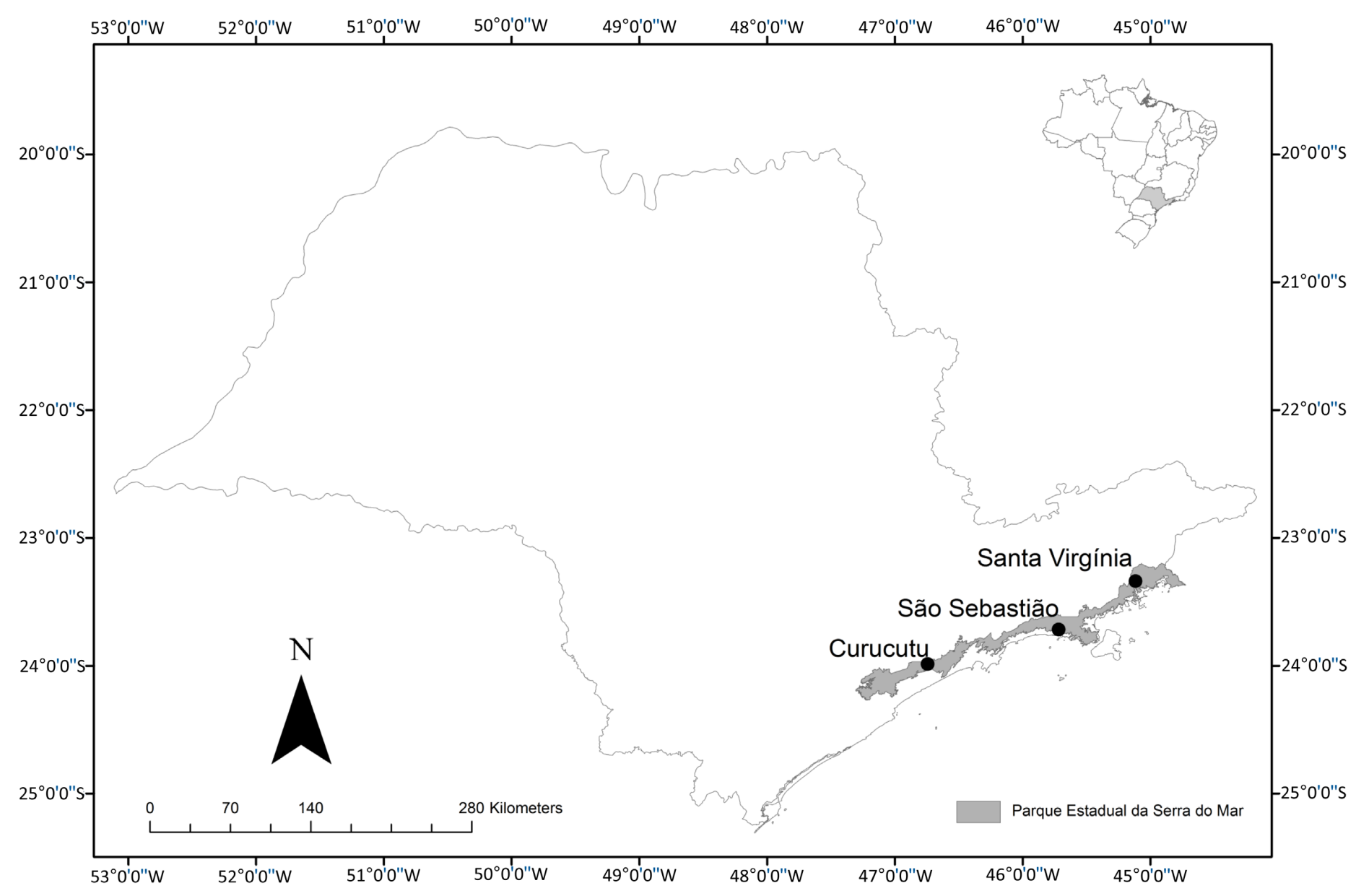

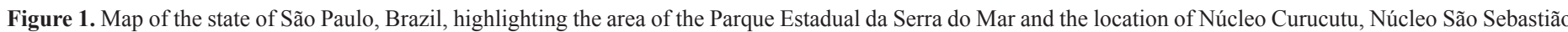
and Núcleo Santa Virginia. 


\section{Survey techniques}

In each of the three localities we sampled amphibian species in two ponds, two streams and two trails inside forest fragments (Table 1, Figure 2) from December 2014 to February 2015, December 2015 to February 2016 and December 2016 to February 2017 totalizing 27 days of field samplings in each sampling unit. We selected this period because is the time of the year when most of annual rainfall occurs and most anuran species are active. We sought to choose sampling units with similar size area and physiognomic characteristics in the three núcleos (Figure 2). We carried out the surveys on streams and trails inside forest fragments considering always the same extension of $100 \mathrm{~m}$ in all núcleos. Furthermore, we selected trails in which there were no water bodies along the extension sampled. We used three methods to record the presence of anurans in each locality: (i) surveys at breeding sites (Scott \& Woodward 1994) - we recorded calling males from 19.00 hours to 24.00 hours; (ii) survey of larvae with dipnetting - we used a long and wire hand net ( $3 \mathrm{~mm}^{2}$ mesh size) along the margins of ponds and streams, sampling the available microhabitats from 12.00 hours to 18.00 hours; and (iii) visual encounter (Crump \& Scott Jr. 1994) - we walked slowly for 30 minutes in trails inside the forest fragment, streams and around ponds looking at microhabitats for individuals hidden under trunks, bromeliads, stones, branches, and leaf litter. All collected specimens (Table 2) were anesthetized and killed, fixed in $10 \%$ formaldehyde and stored in $70 \%$ ethanol (adults) or $10 \%$ formaldehyde (tadpoles). We also collected tissue samples that were fixed in $100 \%$ ethanol and maintained in $-20^{\circ} \mathrm{C}$ in freezers (Table 2). All adult specimens and tissues samples are housed in the Coleção de Anfíbios Célio F. B. Haddad (CFBH), Departamento de Zoologia, Universidade Estadual Paulista, Rio Claro and Coleção de Anfíbios do Departamento de Zoologia e Botânica (DZSJRP), Universidade Estadual Paulista, São José do Rio Preto (Appendix 1). All collected tadpoles are housed in the

Table 1. Geographical coordinates of sample units used in the survey of anurans in Núcleo Curucutu, Núcleo São Sebastião and Núcleo Santa Virgínia of the Parque Estadual da Serra do Mar, state of São Paulo, southeastern Brazil. Geographical coordinates in degrees, minutes and seconds, based on the WGS84 datum.

\begin{tabular}{|c|c|c|c|}
\hline SITES & $\begin{array}{l}\text { LATITUDE } \\
\text { (S) }\end{array}$ & $\begin{array}{c}\text { LONGITUDE } \\
(\mathrm{W})\end{array}$ & $\begin{array}{l}\text { ALTITUDE } \\
(\mathrm{m})\end{array}$ \\
\hline \multicolumn{4}{|c|}{ NÚCLEO CURUCUTU } \\
\hline POND 1 & $23^{\circ} 58^{\prime} 50.69^{\prime \prime}$ & $46^{\circ} 44^{\prime} 08.32^{\prime \prime}$ & 795 \\
\hline POND 2 & $23^{\circ} 59^{\prime} 13.90^{\prime \prime}$ & $46^{\circ} 44^{\prime} 34.43^{\prime \prime}$ & 794 \\
\hline STREAM 1 & $23^{\circ} 59^{\prime} 20.82^{\prime \prime}$ & $46^{\circ} 44^{\prime} 44.09^{\prime \prime}$ & 801 \\
\hline STREAM 2 & $23^{\circ} 59^{\prime} 17.76^{\prime \prime}$ & $46^{\circ} 44^{\prime} 56.64^{\prime \prime}$ & 793 \\
\hline TRAIL 1 & $23^{\circ} 59^{\prime} 06.78^{\prime \prime}$ & $46^{\circ} 44^{\prime} 35.52^{\prime \prime}$ & 788 \\
\hline TRAIL 2 & $23^{\circ} 59^{\prime} 17.04^{\prime \prime}$ & $46^{\circ} 44^{\prime} 36.89^{\prime \prime}$ & 796 \\
\hline \multicolumn{4}{|l|}{$\begin{array}{l}\text { NÚCLEO SÃO } \\
\text { SEBASTIÃO }\end{array}$} \\
\hline POND 1 & $23^{\circ} 44^{\prime} 01.13^{\prime \prime}$ & $45^{\circ} 45^{\prime} 29.31^{\prime \prime}$ & 21 \\
\hline POND 2 & $23^{\circ} 43^{\prime} 08.15^{\prime \prime}$ & $45^{\circ} 43^{\prime} 09.73^{\prime \prime}$ & 72 \\
\hline STREAM 1 & $23^{\circ} 43^{\prime} 03.78^{\prime \prime}$ & $45^{\circ} 43^{\prime} 05.81^{\prime \prime}$ & 71 \\
\hline STREAM 2 & $23^{\circ} 43^{\prime} 01.70^{\prime \prime}$ & $45^{\circ} 42^{\prime} 11.29^{\prime \prime}$ & 335 \\
\hline TRAIL 1 & $23^{\circ} 42^{\prime} 48.37^{\prime \prime}$ & $45^{\circ} 43^{\prime} 34.46^{\prime \prime}$ & 205 \\
\hline TRAIL 2 & $23^{\circ} 43^{\prime} 01.27^{\prime \prime}$ & $45^{\circ} 42^{\prime} 09.47^{\prime \prime}$ & 335 \\
\hline \multicolumn{4}{|l|}{$\begin{array}{l}\text { NÚCLEO SANTA } \\
\text { VIRGÍNIA }\end{array}$} \\
\hline POND 1 & $23^{\circ} 19^{\prime} 55.80^{\prime \prime}$ & $45^{\circ} 5^{\prime} 49.49^{\prime \prime}$ & 979 \\
\hline POND 2 & $23^{\circ} 21^{\prime} 03.93^{\prime \prime}$ & $45^{\circ} 8^{\prime} 07.09^{\prime \prime}$ & 964 \\
\hline STREAM 1 & $23^{\circ} 19^{\prime} 52.14^{\prime \prime}$ & $45^{\circ} 5^{\prime} 51.00^{\prime \prime}$ & 981 \\
\hline STREAM 2 & $23^{\circ} 20^{\prime} 36.96^{\prime \prime}$ & $45^{\circ} 7^{\prime} 45.12^{\prime \prime}$ & 938 \\
\hline TRAIL 1 & $23^{\circ} 20^{\prime} 14.39^{\prime \prime}$ & $45^{\circ} 8^{\prime} 07.14^{\prime \prime}$ & 1000 \\
\hline TRAIL 2 & $23^{\circ} 20^{\prime} 40.44^{\prime \prime}$ & $45^{\circ} 7^{\prime} 58.44^{\prime \prime}$ & 951 \\
\hline
\end{tabular}

Laboratório de Ecologia Teórica: Integrando Biologia, Espaço e Tempo (LET.IT.BE), Departamento de Ciências Ambientais, Universidade Federal de São Carlos, campus Sorocaba (Appendix 1).

\section{Data Analysis}

To assess the sampling efficiency of surveys separately for each locality, we used species accumulation curve (Gotelli \& Colwell 2001) generated from the data of incidence of anurans during the inventory period. The total number of species recorded each day was considered as a sample, totaling 27 samples. Considering the diverse richness estimators available, we chose to use the Jackknife algorithm first order based on its performance when compared to other estimators (Magurran 2004, Walther \& Moore 2005, Hortal et al. 2006). All analyzes were performed in R v 3.2.2 (R Core Team 2015 ) with 1,000 randomizations using vegan (Oksanen et al. 2016) and BiodiversityR (Kindt \& Coe 2005) packages. Taxonomic nomenclature follows Frost (2017). The species conservation status was obtained from The International Union for Conservation of Nature Red List of Threatened Species (IUCN 2016), Brazil Red Book of Threatened Species of Fauna (ICMBio 2015) and Faunal Species of São Paulo state Threatened with Extinction (Garcia et al. 2009).
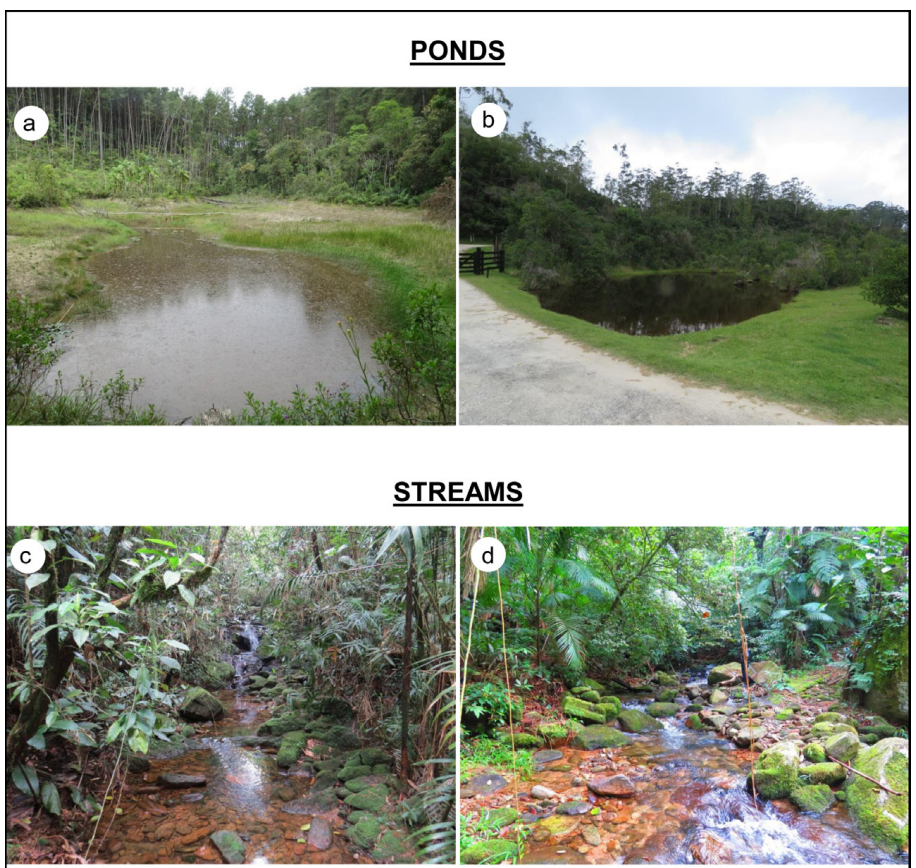

TRAILS INSIDE FOREST FRAGMENTS

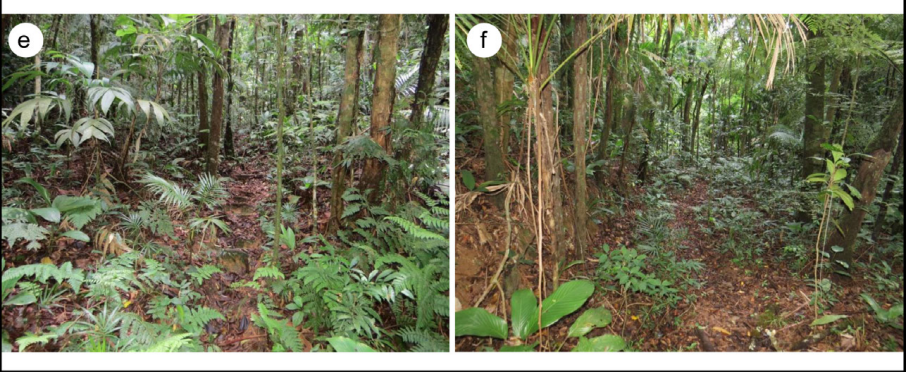

Figure 2. Photographies of ponds (a-b), streams (c-d) and trails inside forest fragments (e-f) used as sample units in the survey of anurans in the núcleos Curucutu, São Sebastião and Santa Virgínia of the Parque Estadual da Serra do Mar, state of São Paulo, southeastern Brazil. 
Table 2. Anuran species composition and abundance from Núcleo Curucutu (PECU), Núcleo São Sebastião (PESB) and Núcleo Santa Virgínia (PESV) of the Parque Estadual da Serra do Mar, state of São Paulo, southeastern Brazil. STATUS IUCN = threatened species categories used in the IUCN Red List Data (IUCN 2016); STATUS BRAZIL $=$ categories of threatened species used in the Brazil Red Book of Threatened Species of Fauna (ICMBio 2015); STATUS SÃO PAULO $=$ categories of threatened species used in the São Paulo Red Book of Threatened Species of Fauna (Garcia et al. 2009): DD (Data Deficient), LC (Least Concern) and CE (Critically Endangered). POP. TREND = current population trends used in IUCN Red List Data: increasing, decreasing, stable or unknown. $*=$ species not evaluated or not cited. \# Cryptic species: in this case, abundance was defined as only one species in the fieldwork.

\section{Family/Species}

PECU PESB PESV

$\begin{array}{cc}\text { STATUS } & \text { STATUS } \\ \text { IUCN } & \text { BRAZIL }\end{array}$

\begin{tabular}{cc} 
STATUS & POP. \\
SÃO & TREND \\
PAULO & \\
\hline
\end{tabular}

\section{BUFONIDADE}

Dendrophryniscus brevipollicatus Jiménez de la Espada, 1870

Dendrophryniscus sp.

Rhinella hoogmoedi Caramaschi \& Pombal, 2006

Rhinella icterica (Spix, 1824)

Rhinella ornata (Spix, 1824)

BRACHYCEPHALIDAE

Brachycephalus pitanga Alves, Reis \& Haddad, 2009

Ischnocnema gr. guentheri (CS1 lineage, Gehara et al. 2013)

Ischnocnema gr. guentheri (CS3 lineage, Gehara et al. 2013)

Ischnocnema gr. guentheri (CS4 lineage, Gehara et al. 2013)

Ischnocnema henselii (Peters, 1870)

Ischnocnema parva (Girard, 1853)

\section{CENTROLENIDAE}

Vitreorana uranoscopa (Müller, 1924)

CRAUGASTORIDAE

Haddadus binotatus (Spix, 1824)

CYCLORAMPHIDAE

Cycloramphus boraceiensis Heyer, 1983

Thoropa taophora (Miranda-Ribeiro, 1923)

HEMIPHRACTIDAE

Fritziana sp. (aff. fissilis)

HYLIDAE

Aplastodiscus albofrenatus (Lutz, 1924)

Aplastodiscus leucopygius (Cruz \& Peixoto, 1985)

Bokermannohyla izecksohni (Jim \& Caramaschi, 1979)

Bokermannohyla circumdata (Cope, 1871)

Bokermannohyla hylax (Heyer, 1985)

Dendropsophus berthalutzae (Bokermann, 1962)

Dendropsophus elegans (Wied-Neuwied, 1824)

Dendropsophus microps (Peters, 1872)

Dendropsophus minutus (Peters, 1872)

Dendropsophus seniculus (Cope, 1868)

Boana albomarginata (Spix, 1824)

Boana albopunctata (Spix, 1824)

Boana bandeirantes (Caramaschi \& Cruz, 2013)

Boana bischoffi (Boulenger, 1887)

Boana faber (Wied-Neuwied, 1821)

Boana pardalis (Spix, 1824)

Boana semilineata (Spix, 1824)

Ololygon argyreornata (Miranda-Ribeiro, 1926)

Ololygon littoralis (Pombal \& Gordo, 1991)

Ololygon cf. obtriangulata (Lutz, 1973)

Ololygon perpusilla (Lutz \& Lutz, 1939)

Ololygon rizibilis (Bokermann, 1964)
8

1

16

11

14

12

24

5

20

7

25

22

1

12

28

52

1

1$$
2
$$

12

4

25

2

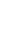

1

2

1

5

5$$
28
$$

1

1

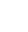

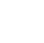

6

.

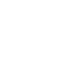

(20)

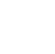

2

1

$5 \quad 20$

4

$\begin{array}{cccc}\text { LC } & \text { LC } & \text { LC } & \text { Stable } \\ * & * & * & * \\ \text { LC } & \text { LC } & \text { LC } & \text { Unknown } \\ \text { LC } & \text { LC } & \text { LC } & \text { Stable } \\ \text { LC } & \text { LC } & \text { LC } & \text { Stable }\end{array}$

18

$16^{\#}$

$16^{\#}$

43

24

3

LC

LC

*

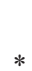

C

CE

LC

LC

LC

LC

$$
\text { LC }
$$

$$
\text { LC }
$$$$
\text { LC }
$$

LC

LC

*

LC

LC

LC

LC

LC

LC

LC

LC

LC

LC

$\begin{array}{cc}* & * \\ * & * \\ * & * \\ * & * \\ * & \text { Unknown } \\ \text { LC } & \text { Decreasing }\end{array}$

LC

Decreasing

LC Stable

LC

Decreasing

LC
LC

DD

LC

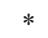

\begin{tabular}{lcc}
$*$ & $*$ & $*$ \\
LC & $*$ & Stable \\
LC & LC & Stable \\
DD & LC & Decreasing \\
LC & LC & Decreasing \\
LC & LC & Decreasing \\
LC & LC & Stable \\
LC & LC & Stable \\
LC & LC & Stable \\
LC & LC & Stable \\
LC & LC & Stable \\
LC & LC & Stable \\
LC & LC & Stable \\
LC & $*$ & $*$ \\
LC & LC & Stable \\
LC & LC & Stable \\
LC & LC & Stable \\
LC & LC & Stable \\
LC & LC & Stable \\
LC & LC & Decreasing \\
LC & LC & Decreasing \\
LC & LC & Stable \\
LC & LC & Decreasing \\
\hline
\end{tabular}


Table 2. Continued...

\begin{tabular}{|c|c|c|c|c|c|c|c|}
\hline Family/Species & PECU & PESB & PESV & $\begin{array}{l}\text { STATUS } \\
\text { IUCN }\end{array}$ & $\begin{array}{l}\text { STATUS } \\
\text { BRAZIL }\end{array}$ & $\begin{array}{c}\text { STATUS } \\
\text { SÃO } \\
\text { PAULO }\end{array}$ & $\begin{array}{c}\text { POP. } \\
\text { TREND }\end{array}$ \\
\hline Scinax crospedospilus (Lutz, 1925) & 1 & 5 & 5 & $\mathrm{LC}$ & $\mathrm{LC}$ & LC & Stable \\
\hline Scinax fuscovarius (Lutz, 1925) & 1 & & 1 & $\mathrm{LC}$ & $\mathrm{LC}$ & $\mathrm{LC}$ & Stable \\
\hline Scinax hayii (Barbour, 1909) & & 10 & 26 & $\mathrm{LC}$ & $\mathrm{LC}$ & $\mathrm{LC}$ & Stable \\
\hline Scinax perereca Pombal, Haddad \& Kasahara, 1995 & 8 & & & $\mathrm{LC}$ & $\mathrm{LC}$ & $\mathrm{LC}$ & Unknown \\
\hline Scinax tymbamirim Nunes, Kwet \& Pombal, 2012 & 36 & 22 & 5 & $*$ & $*$ & $*$ & $*$ \\
\hline Trachycephalus mesophaeus (Hensel, 1867) & & 1 & & $\mathrm{LC}$ & $\mathrm{LC}$ & $\mathrm{LC}$ & Decreasing \\
\hline Trachycephalus imitatrix (Miranda-Ribeiro, 1926) & & & 2 & $\mathrm{LC}$ & $\mathrm{LC}$ & $\mathrm{DD}$ & Decreasing \\
\hline \multicolumn{8}{|l|}{ HYLODIDAE } \\
\hline Hylodes asper (Müller, 1924) & & 15 & & $\mathrm{LC}$ & $\mathrm{LC}$ & $\mathrm{LC}$ & Stable \\
\hline Hylodes phyllodes Heyer \& Crocoft, 1986 & & 15 & 10 & $\mathrm{LC}$ & $\mathrm{LC}$ & $\mathrm{LC}$ & Decreasing \\
\hline Megaelosia cf. bocainensis Giaretta, Bokermann \& Haddad, 1993 & & & 1 & DD & DD & DD & Unknown \\
\hline \multicolumn{8}{|l|}{ LEPTODACTYLIDAE } \\
\hline Adenomera ajurauna (Berneck, Costa \& Garcia, 2008) & 10 & & & DD & $*$ & $\mathrm{LC}$ & Stable \\
\hline Adenomera marmorata Steindachner, 1867 & 20 & 70 & 32 & $\mathrm{LC}$ & $\mathrm{LC}$ & $\mathrm{LC}$ & Stable \\
\hline Adenomera sp. & & 15 & & $*$ & $*$ & $*$ & * \\
\hline Leptodactylus furnarius Sazima \& Bokermann, 1978 & 24 & & & $\mathrm{LC}$ & $\mathrm{LC}$ & $\mathrm{LC}$ & Stable \\
\hline Leptodactylus fuscus (Schneider, 1799) & & & 3 & $\mathrm{LC}$ & $\mathrm{LC}$ & $\mathrm{LC}$ & Stable \\
\hline Leptodactylus plaumanni Ahl, 1936 & 3 & & & $\mathrm{LC}$ & $\mathrm{LC}$ & $*$ & Stable \\
\hline Leptodactylus latrans (Steffen, 1815) & 7 & 1 & 1 & $\mathrm{LC}$ & $\mathrm{LC}$ & $\mathrm{LC}$ & Stable \\
\hline Paratelmatobius cardosoi Pombal \& Haddad, 1999 & 13 & & & DD & $\mathrm{LC}$ & $\mathrm{LC}$ & Unknown \\
\hline Physalaemus atlanticus Haddad \& Sazima, 2004 & & 10 & & $\mathrm{VU}$ & $\mathrm{LC}$ & $\mathrm{LC}$ & Unknown \\
\hline Physalaemus bokermanni Cardoso \& Haddad, 1985 & & 20 & & DD & $\mathrm{LC}$ & $\mathrm{LC}$ & Decreasing \\
\hline Physalaemus cuvieri Fitzinger, 1826 & 25 & & 7 & $\mathrm{LC}$ & $\mathrm{LC}$ & $\mathrm{LC}$ & Stable \\
\hline Physalaemus olfersii (Lichtenstein \& Martens, 1856) & & 3 & 14 & $\mathrm{LC}$ & $\mathrm{LC}$ & $\mathrm{LC}$ & Stable \\
\hline \multicolumn{8}{|l|}{ MICROHYLIDAE } \\
\hline Chiasmocleis leucosticta (Boulenger, 1888) & & 2 & & $\mathrm{LC}$ & $\mathrm{LC}$ & $\mathrm{LC}$ & Stable \\
\hline Myersiella microps (Duméril \& Bibron, 1841) & & 4 & & $\mathrm{LC}$ & LC & $\mathrm{LC}$ & Stable \\
\hline \multicolumn{8}{|l|}{ ODONTOPHRYNIDAE } \\
\hline Proceratophrys boiei (Wied-Neuwied, 1824) & & 2 & 2 & $\mathrm{LC}$ & $\mathrm{LC}$ & $\mathrm{LC}$ & Stable \\
\hline Proceratophrys melanopogon (Miranda-Ribeiro, 1926) & & 4 & & $\mathrm{LC}$ & $\mathrm{LC}$ & $\mathrm{LC}$ & Decreasing \\
\hline \multicolumn{8}{|l|}{ PHYLLOMEDUSIDAE } \\
\hline Phrynomedusa dryade Baêta, Giasson, Pombal \& Haddad, 2016 & & & 10 & * & * & * & * \\
\hline Number of Species & 34 & 44 & 42 & & & & \\
\hline
\end{tabular}

\section{Results and Discussion}

We recorded 34 species in Núcleo Curucutu, 44 species in Núcleo São Sebastião and 42 species in Núcleo Santa Virgínia (Tabela 2, Figures 3-6), totalizing 65 anuran species belonging to 12 families (number in parentheses): Bufonidae (5), Brachycephalidae (6), Centrolenidae (1), Craugastoridae (1), Cycloramphidae (2), Hemiphractidae (1), Hylidae (29), Hylodidae (3), Leptodactylidae (12), Microhylidae (2), Odontophrynidae (2) and Phyllomedusidae (1). From the 65 species recorded, 20 species $(30.7 \%)$ occurred in the three núcleos, 14 species $(21.5 \%)$ occurred in two núcleos, eight species (12.3\%) occurred only at Núcleo Curucutu, nine species (13.8\%) occurred only at Núcleo Santa Virgínia and 14 species (21.5\%) occurred only at Núcleo São Sebastião (Table 2). The observed species richness was smaller than the estimated species richness (Figure 7), indicating that 4.8 more species could be recorded at núcleos Curucutu, 6.7 more species at Núcleo Santa Virgínia and 5.7 more species at the
Núcleo São Sebastião if we increase the sampling effort or utilize other sampling methods (e.g., pitfall traps). For example, Malagoli (2008, 2013) using data gathered from fieldwork, scientific collection and grey literature recorded 66 anuran species to Núcleo Curucutu. Giasson (2008) carried out 25 excursions to Núcleo Santa Virgínia from January 2003 to December 2006 and recorded 50 anuran species. However, we are aware that these studies used different sampling efforts and their results are not comparable to species richness observed in our study.

According to the threatened species categories used in IUCN (2016), Bokermannohyla izecksohni is classified as critically endangered, Physalaemus atlanticus is classified as vulnerable, Adenomera ajurauna, Megaelosia $\mathrm{cf}$. bocainensis, Paratelmatobius cardosoi and P. bokermanni are classified as data deficient and other anuran species are classified as least concern (Table 2).On the other hand, considering threatened species categories used in the Brazilian (ICMBio 2015) and state of São Paulo (Garcia et al. 2009) lists, B. izecksohni, Cycloramphus boraceiensis, Trachycephalus imitatrix 

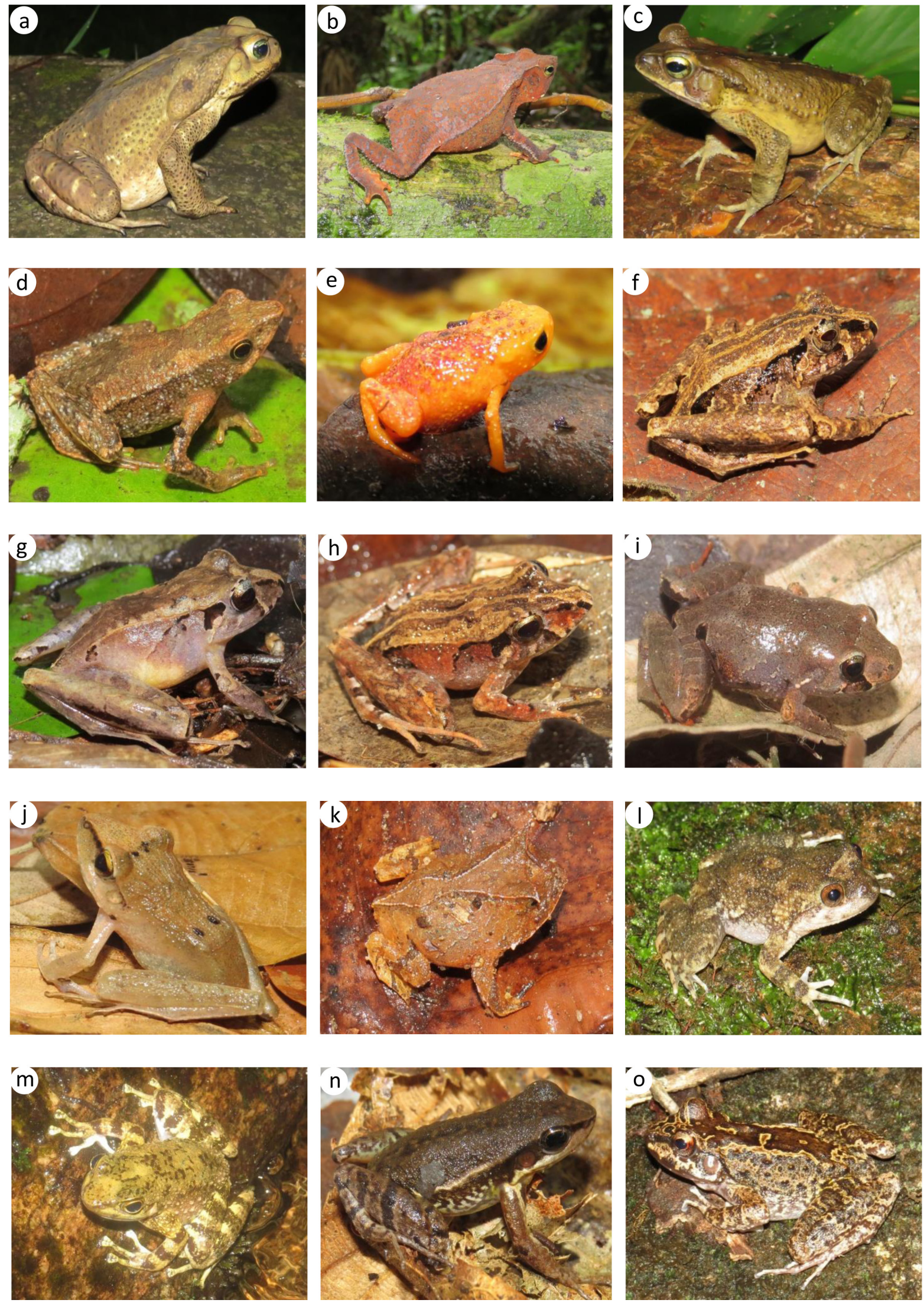

Figure 3. Anuran species recorded at the Parque Estadual da Serra do Mar, Núcleo Curucutu, Núcleo São Sebastião and Núcleo Santa Virgínia, state of São Paulo, southeastern Brazil. $\mathrm{a}=$ Rhinella icterica, $\mathrm{b}=R$. hoogmoedi, $\mathrm{c}=R$. ornata, $\mathrm{d}=$ Dendrophryniscus brevipollicatus, $\mathrm{e}=$ Brachycephalus pitanga, $\mathrm{f}=$ Ischnocnema gr. guentheri (CS1 lineage Gehara et al. 2013), g = Ischnocnema gr. guentheri (CS4 lineage Gehara et al. 2013), $\mathrm{h}=$ I. henselii, $\mathrm{i}=I$. parva, $\mathrm{j}=$ Haddadus binotatus, $\mathrm{k}=$ Proceratophrys melanopogon, $\mathrm{l}=$ Cycloramphus boraceiensis, $\mathrm{m}=$ Hylodes asper, $\mathrm{n}=$ H. phyllodes and $\mathrm{o}=$ Thoropa taophora . 

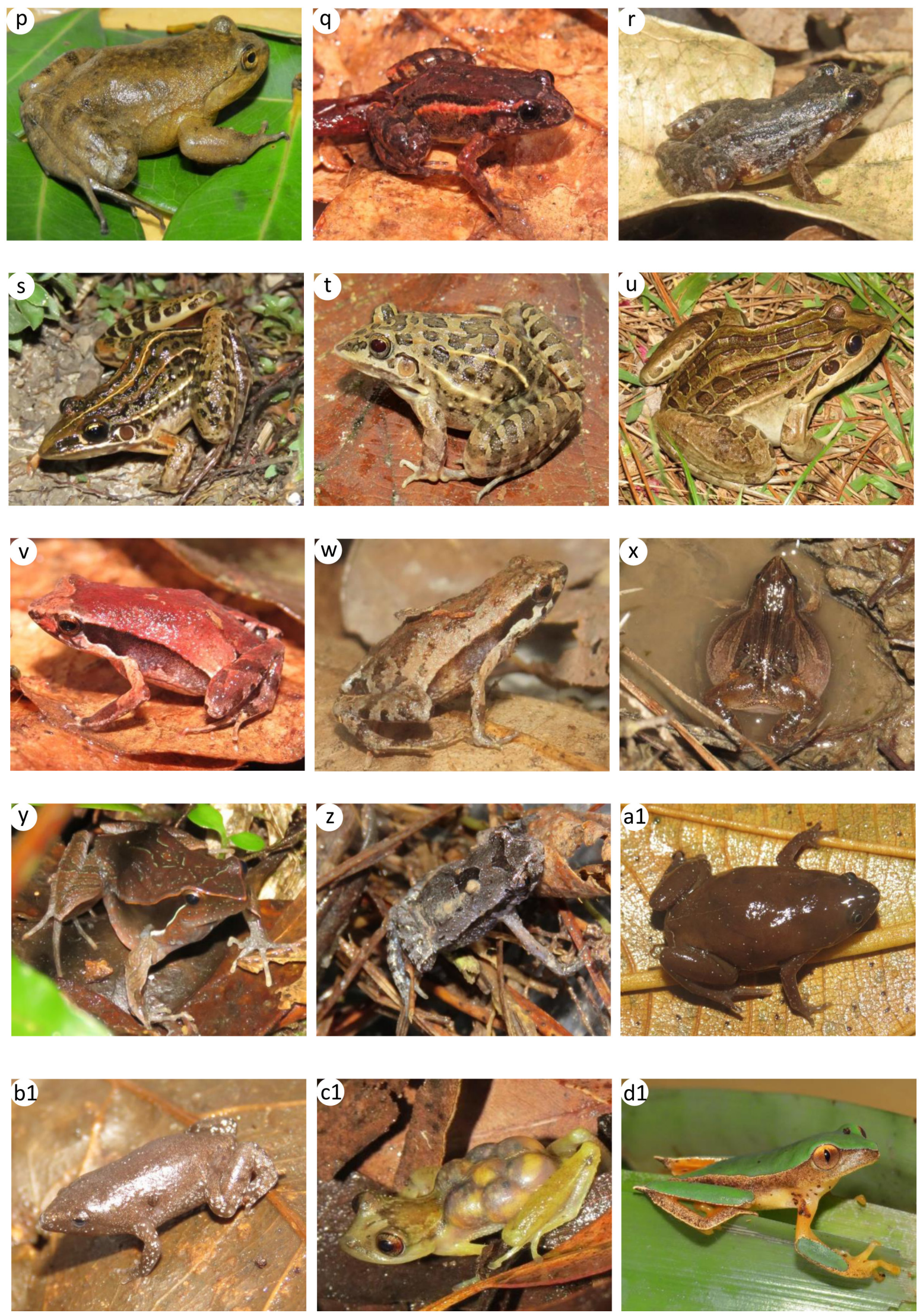

Figure 4. Anuran species recorded at the Parque Estadual da Serra do Mar, Núcleo Curucutu, Núcleo São Sebastião and Núcleo Santa Virgínia, state of São Paulo, southeastern Brazil. $\mathrm{p}=$ Megaelosia $\mathrm{cf}$. bocainensis, $\mathrm{q}=$ Adenomera marmorata, $\mathrm{r}=$ Adenomera $\mathrm{sp} ., \mathrm{s}=$ Leptodactylus furnarius, $\mathrm{t}=$ L. fuscus, $\mathrm{u}=$ L. latrans, $\mathrm{v}=$ Physalaemus atlanticus, $\mathrm{w}=$ P. bokermanni, $\mathrm{x}=P$. cuvieri, $\mathrm{y}=$ P. olfersii, $\mathrm{z}=$ Paratelmatobius cardosoi, $\mathrm{a} 1=$ Chiasmocleis leucosticta, $\mathrm{b} 1=$ Myersiela microps, $\mathrm{c} 1=$ Fritziana $\mathrm{sp}$. (aff. fissilis) and d1 = Phrynomedusa dryade. 

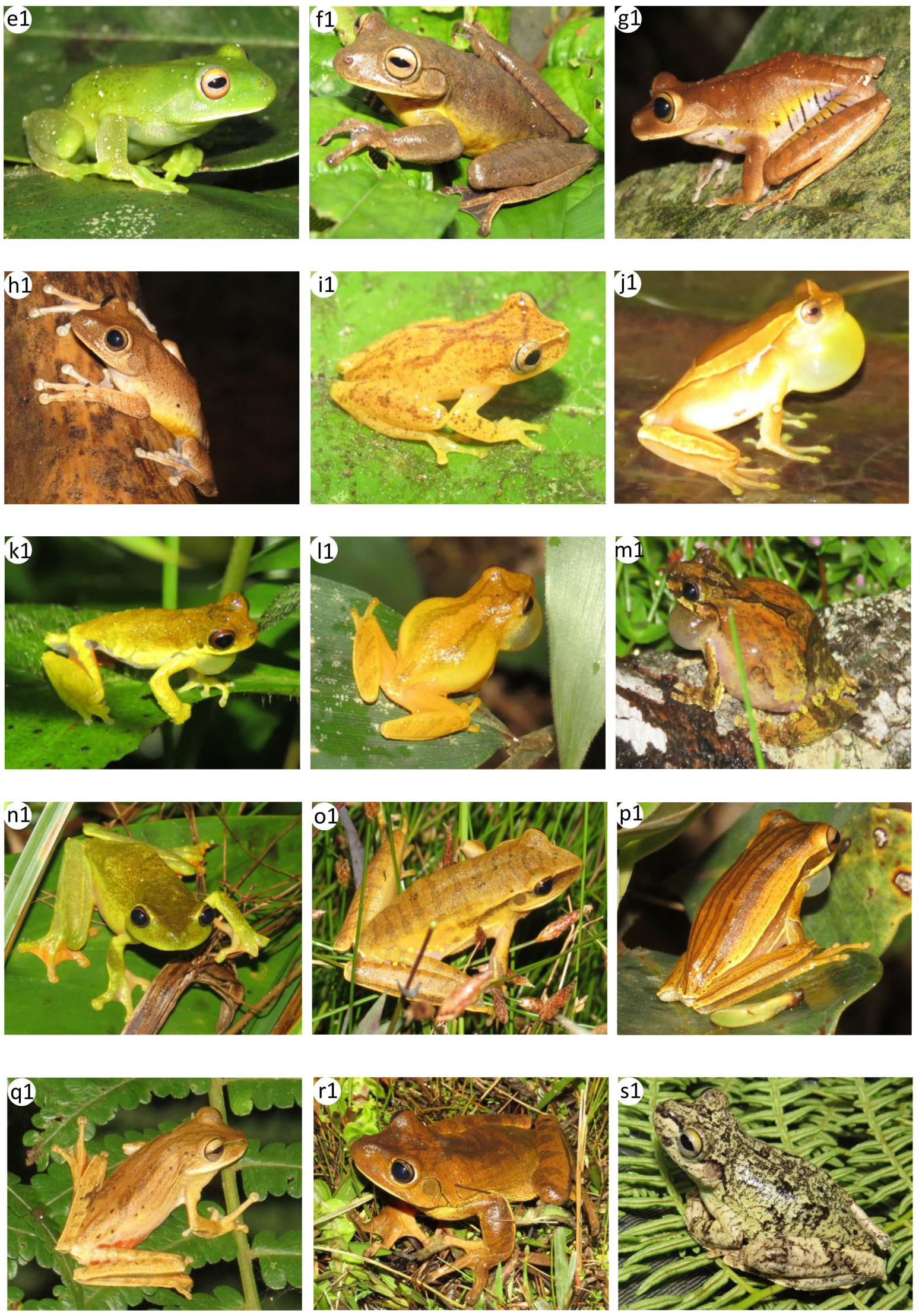

Figure 5. Anuran species recorded at the Parque Estadual da Serra do Mar, Núcleo Curucutu, Núcleo São Sebastião and Núcleo Santa Virgínia, state of São Paulo, southeastern Brazil. $\mathrm{e} 1=$ Aplastodiscus leucopygius, $\mathrm{f1}=$ Bokermannohyla circumdata, $\mathrm{g} 1=B$. hylax, $\mathrm{h} 1=B$. izecksohni, $\mathrm{i} 1=$ Dendropsophus berthalutzae, $\mathrm{j} 1=$ D. elegans, $\mathrm{k} 1=$ D. microps, $11=$ D. minutus, $\mathrm{m} 1=D$. seniculus, $\mathrm{n} 1=$ Boana albomarginata, $\mathrm{o} 1=B$. albopunctata, $\mathrm{p} 1=B$. bandeirantes, $\mathrm{q} 1=B$. bischoffi, $\mathrm{r} 1=B$. faber and $\mathrm{s} 1=$ B. pardalis. 

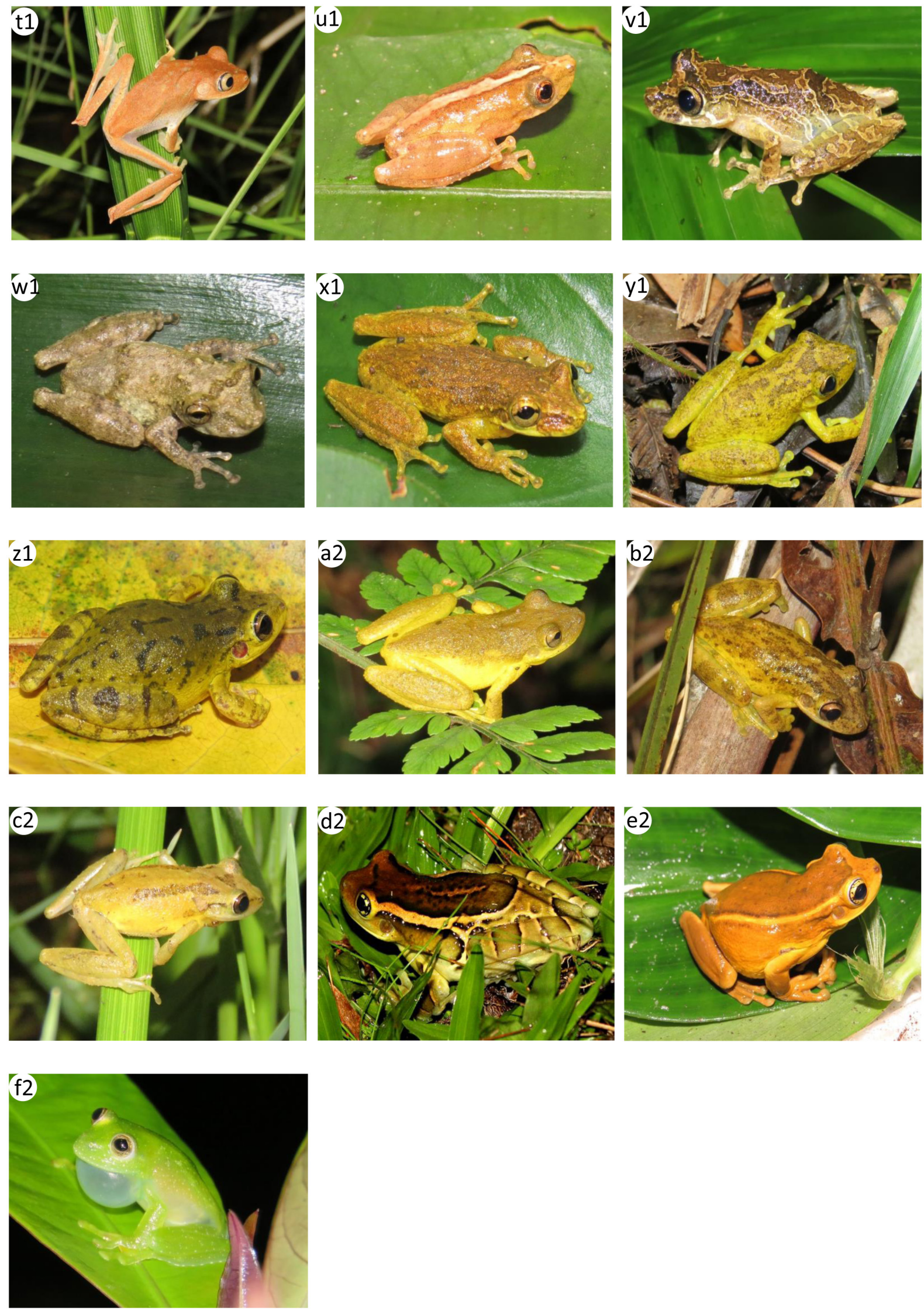

Figure 6. Anuran species recorded at the Parque Estadual da Serra do Mar, Núcleo Curucutu, Núcleo São Sebastião and Núcleo Santa Virgínia, state of São Paulo, southeastern Brazil. $\mathrm{t} 1=$ B. semilineata, $\mathrm{u} 1=$ Ololygon argyreornata, $\mathrm{v} 1=$ O. littoralis, $\mathrm{w} 1=O$. perpusilla, $\mathrm{x} 1=O$. rizibilis, $\mathrm{y} 1=S$ cinax crospedospilus, $\mathrm{z} 1=S$. fuscovarius, $\mathrm{a} 2=$ S. hayii, $\mathrm{b} 2=$ S. perereca, $\mathrm{c} 2=$ S. tymbamirim, $\mathrm{d} 2=$ Trachycephalus imitatrix, $\mathrm{e} 2=$ T. mesophaeus and $\mathrm{f} 2=$ Vitreorana uranoscopa. 


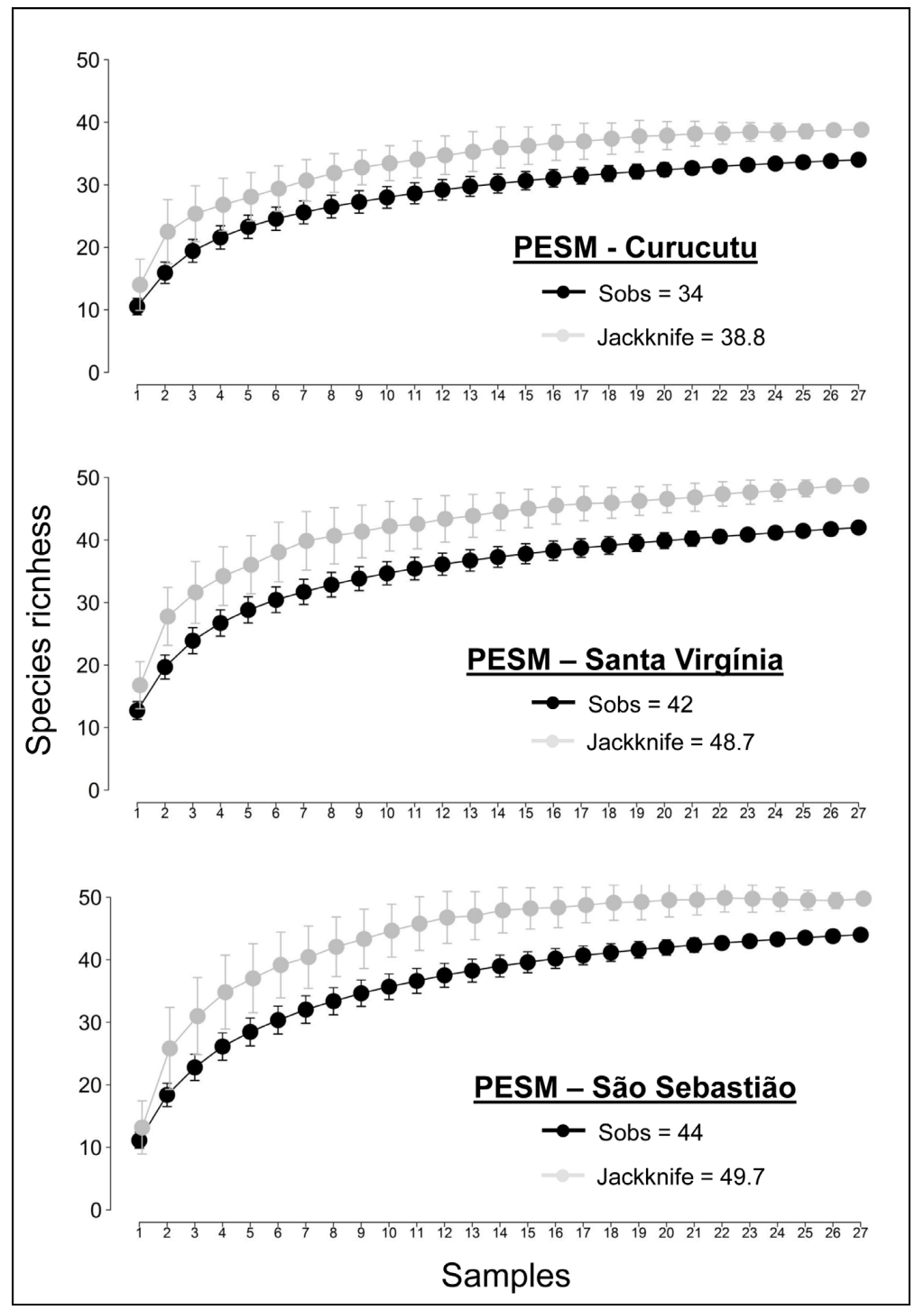

Figure 7. Species accumulation curve (black line) and Jackknife algorithm first order (gray line) representing the anuran species richness observed and estimated, respectively, based on 18 samples from December 2014 to February 2017 at the Parque Estadual da Serra do Mar, Núcleo Curucutu, Núcleo São Sebastião and Núcleo Santa Virgínia, state of São Paulo, southeastern Brazil. The dots show the mean generated by 1000 randomizations and the vertical bars indicate the standard deviation.

and Megaelosia cf. bocainensis are classified as data deficient and other anuran species are classified as least concern (Table 2). Considering the trend populations of IUCN (2016), 14 species have declining populations (Ischnocnema parva, Vitreorana uranoscopa, C. boraceiensis, B. izecksohni, B. circumdata, B. hylax, Ololygon littoralis, Ololygon cf. obtriangulata, O. rizibilis, T. imitatrix, T. mesophaeus, Hylodes phyllodes, P. bokermanni and Proceratophrys melanopogon), and other six species have unknown population trends (I. henselli, Scinax perereca, Megaelosia $\mathrm{cf}$. bocainensis, P. cardosoi, P. atlanticus and Rhinella hoogmoedi) (Table 2). It is important to recognize that IUCN trend populations it is not consensual among researchers and it may contain imprecisions. For example, the populations of widespread species with recognized tolerance to anthropogenic habitats such as T. mesophaeus probably may not be declining (Carvalho-e-Silva \& Garcia 2004).

We registered 1282 individuals being that Ololygon perpusillus and Boana albopunctata were the most abundant species at Núcleo Curucutu (Figure 8B), Ischnocnema parva and Dendropsophus microps were the most abundant species at Núcleo Santa Virgínia (Figure 8C) and
Adenomera marmorata and Ololygon littoralis were the most abundant species at Núcleo São Sebastião (Figure 8D). Most anuran species were recorded more than once during the sampling period; however, Thoropa taophora, B. izecksohni, O. argyreornata, Ololygon cf. obtriangulata, T. mesophaeus, Megaelosia cf. bocainensis and R. hoogmoedi were recorded on only one occasion based on a single individual (Figure 8A). Except for Megaelosia $\mathrm{cf}$. bocainensis whose natural history and population trend are unknown, Thoropa taophora, B. izecksohni, O. argyreornata, Ololygon cf. obtriangulata, T. mesophaeus and $R$. hoogmoedi are common species that reproduce during one or few days along the year (i.e. explosive breeding). Therefore, the low abundance recorded for these species probably is due to sampling effort. Information about species richness and composition are the basic dataset for ecology, systematics, biogeography and conservation biology (e.g., Collen et al. 2008, Da Silva et al. 2012, 2014) as well as they are relevant to subsidize management plans and conservation strategies. Therefore, we hope that these lists can be useful for future studies as well as helping in the management and conservation planning of these protected areas. 

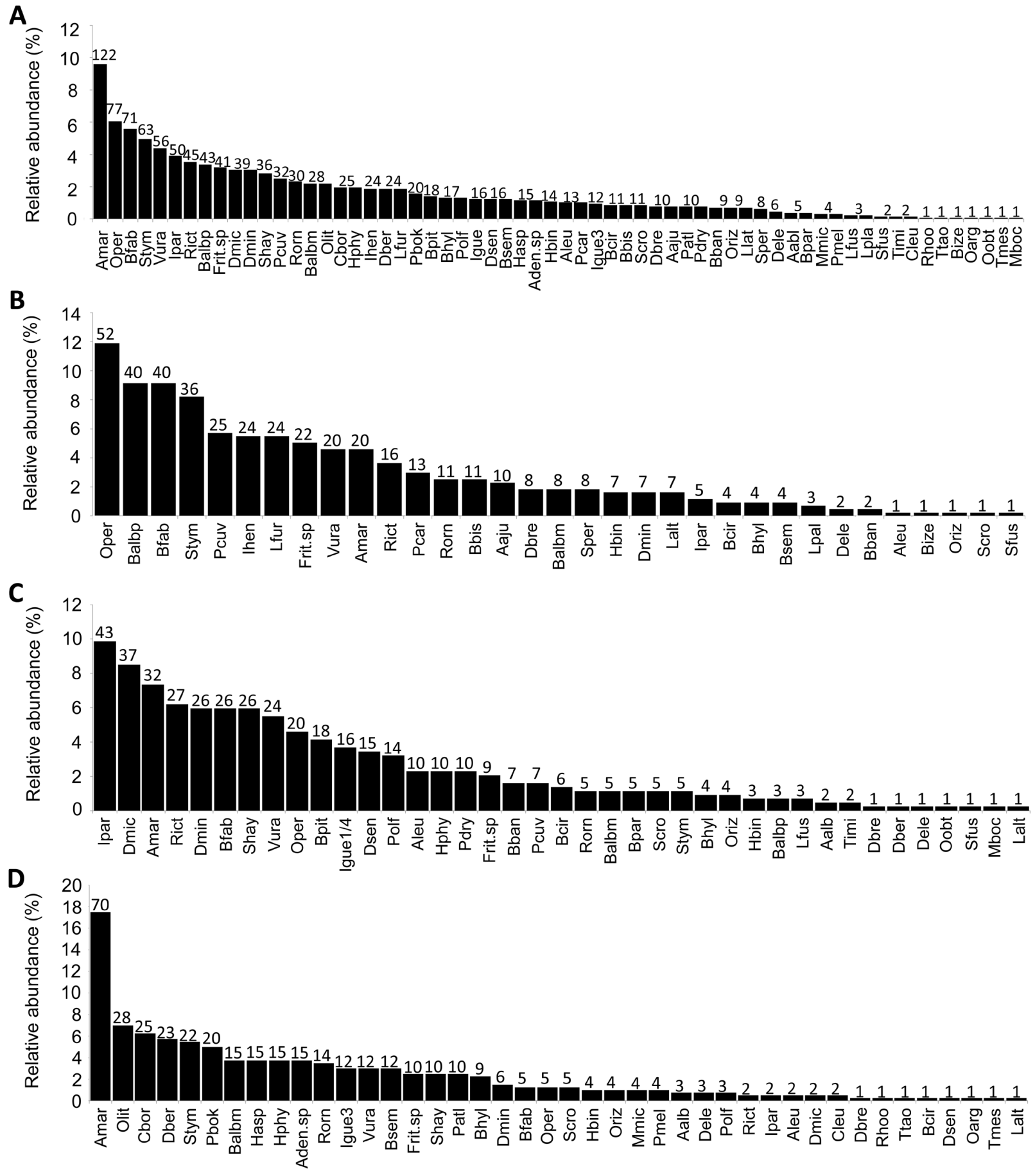

Figure 8. Relative abundance of anuran species (\%) observed at the Parque Estadual da Serra do Mar, state of São Paulo, southeastern Brazil. (A) sum of all individuals observed at the Núcleo Curucutu, Núcleo Curucutu and Núcleo Santa Virgínia; (B) individuals observed only at the Núcleo Santa Virgínia; (C) individuals observed only at the Núcleo São Sebastião; (D) individuals observed only at the Núcleo São Sebastião. The total number of recorded individuals per species is indicated above each column. Anuran species: Rict, Rhinella icterica; Rhoog, R. hoogmoedi; Rorn, R. ornata; Dbre, Dendrophryniscus brevipollicatus; Bpit, Brachycephalus pitanga; Igue1, Ischnocnema gr. guentheri (CS1 lineage Gehara et al. 2013); Igue3, I. gr. guentheri (CS3 lineage Gehara et al. 2013); Igue4, I. gr. guentheri (CS4 lineage Gehara et al. 2013); Ihen, I. henselii; Ipar, I. parva, Hbin, Haddadus binotatus; Pmel, Proceratophrys melanopogon; Cbor, Cycloramphus boraceiensis; Hasp, Hylodes asper; Hphy, H. phyllodes; Ttao, Thoropa taophora; Mboc, Megaelosia cf. bocainensis; Amar, Adenomera marmorata; Aden.sp, Adenomera sp.; Lfur, Leptodactylus furnarius; Lfus, L. fuscus; Llat, L. latrans; Patl, Physalaemus atlanticus; Pbok, P. bokermanni; Pcuv, P. cuvieri; Polf, P. olfersii; Pcar, Paratelmatobius cardosoi; Cleu, Chiasmocleis leucosticta; Mmic, Myersiela microps; Frit.sp, Fritziana sp. (aff. fissilis); Pdry, Phrynomedusa dryade; Aleu, Aplastodiscus leucopygius; Bcir, Bokermannohyla circumdata; Bhyl, B. hylax; Bize, B. izecksohni; Dber, Dendropsophus berthalutzae; Dele, D. elegans; Dmic, D. microps; Dmin, D. minutus; Dsen, D. seniculus; Balbm, Boana albomarginata; Balbp, B. albopunctata; Bban, B. bandeirantes; Bbis, B. bischoff; Bfab, B. faber; Bpar, B. pardalis; Bsem, B. semilineata; Oarg, Ololygon argyreornata; Olit, O. littoralis; Oper, O. perpusilla; Oriz, O. rizibilis; Scro, Scinax crospedospilus; Sfus, S. fuscovarius; Shay, S. hayii; Sper, S. perereca; Stym, S. tymbamirim; Timi, Trachycephalus imitatrix; Tmes, T. mesophaeus; Vura, Vitreorana uranoscopa. 


\section{Supplementary material}

The following online material is available for this article:

Appendix 1: Vouchers of anurans collected at núcleos Curucutu, São Sebastião and Santa Virgínia of the Parque Estadual da Serra do Mar state of São Paulo, southeastern Brazil. VOUCHER AND TISSUES = register number of the Coleção de Anfíbios Célio F. B. Haddad, Departamento de Zoologia, Universidade Estadual Paulista, Rio Claro (CFBH); Coleção de Anfíbios do Departamento de Zoologia e Botânica, Universidade Estadual Paulista, São José do Rio Preto (DZSJRP); Laboratório de Ecologia Teórica:Integrando Biologia, Espaço e Tempo (LET.IT.BE), Departamento de Ciências Ambientais, Universidade Federal de São Carlos, campus Sorocaba (FRS). * Species recorded by vocalization in the fieldwork.

\section{Acknowledgements}

We thank the Instituto Chico Mendes de Conservação da Biodiversidade (ICMBio) for providing collection permits (SISBIO/41282-1) and the Instituto Florestal de São Paulo for the permission to perform the study in the Parque Estadual da Serra do Mar, núcleos Curucutu, São Sebastião and Santa Virgínia (Proc. 260108-004.608/2014). We thank all the staff of the núcleos Curucutu, São Sebastião and Santa Virgínia for helping us during fieldworks. FRS thanks São Paulo Research Foundation (FAPESP - grant \#2013/50714-0) for financial support. MLL thanks FAPESP and Coordenação de Aperfeiçoamento de Pessoal do Nível Superior (CAPES) for postdoctoral fellowships (grants \#2010/50124-0; 88881.062205/2014-01). CFBH thanks Fundação Grupo Boticário de Proteção à Natureza and FAPESP (grants \#2013/50741-7 and \#2014/50342-8) and Conselho Nacional de Desenvolvimento Científico e Tecnológico (CNPq - Proc. 302518/2013-4) for a research fellowship. DCRF thanks CNPq (Proc. 303522/2013-5).

\section{Author Contributions}

Fernando Rodrigues da Silva: contributed in the acquisition, analysis, data interpretation and writing of the work. Mariana Lúcio Lyra: contributed in the molecular analysis, data interpretation and writing of the work. Célio Fernando Baptista Haddad: contributed in the identification of species, data interpretation and writing of the work. Denise de Cerqueira Rossa-Feres: contributed in the data interpretation and writing of the work.

\section{Conflicts of interest}

The author(s) declare(s) that they have no conflict of interest related to the publication of this manuscript.

\section{References}

CARVALHO-E-SILVA, S.P. \& GARCIA, P. 2004.Trachycephalus mesophaeus. The IUCN Red List of Threatened Species 2004: e.T55822A11372732.http:/ dx.doi.org/10.2305/IUCN.UK.2004.RLTS.T55822A11372732.en (last access in $29 / 03 / 2017)$

COLLEN, B., RAM, M., ZAMIM, T. \& MCRAE, L. 2008. The tropical biodiversity data gap: addressing disparity in global monitoring. Trop. Conserv. Sci. 1: 75-88.

CRUMP, M.L. \& SCOTT Junior, N.J. 1994. Visual encounter surveys. In Measuring and monitoring biological diversity: standard methods for amphibians (W.R Heyer, M.A. Donnelly, R.W. McDiarmid, L.C. Hayek \& M.S Foster, eds) Smithsonian Institution Press, Washington, p.84-92.

DA SILVA, F.R., ALMEIDA-NETO, M., PRADO, V.H.M., HADDAD, C.F.B. \& ROSSA-FERES, D.C. 2012. Humidity levels drive reproductive modes and phylogenetic diversity of amphibians in the Brazilian Atlantic Forest. J. Biogeogr. 39:1720-1732.

DA SILVA, F.R., ALMEIDA-NETO, M., ARENA, M.V.N. 2014. Amphibian beta diversity in the Brazilian Atlantic Forest: contrasting the roles of historical events and contemporary conditions at different spatial scales. PLoS ONE 9(10): e109642. doi:10.1371/journal.pone.0109642

FROST, D.R. 2017. Amphibian species of the world: an Online Reference. Version 6.0. Eletronic Database accessible at http://research.amnh.org/herpetology/ amphibia/index.html. American Museum of Natural History, New York, USA (last access in 10/05/2017).

GARCIA, P.C.A., SAWAYA, R.J., MARTINS, I.A., BRASILEIRO, C.A., VERDADE, V.K., JIM, J., SEGALLA, M.V., MARTINS, M., ROSSA-FERES, D.C., HADDAD, C.F.B., TOLEDO, L.F., PRADO, C.P.A., BERNECK, B.M. \& ARAÚJO, O.G.S. 2009. Anfíbios. In Fauna ameaçada de extinção no Estado de São Paulo, Vertebrados (P.M. Bressan, M.C.M. Kierulff \& A.M. Sugieda, eds). Governo do Estado de São Paulo, Secretaria do Meio Ambiente, Fundação Parque Zoológico de São Paulo, São Paulo, p.329-347.

GEHARA, M., CANEDO, C., HADDAD, C.F.B. \& VENCES, M. 2013. From widespread to microendemic: molecular and acoustic analyses show that Ischnocnema guentheri (Amphibia: Brachycephalidae) is endemic to Rio de Janeiro, Brazil. Conserv. Genet. 14: 973-982.

GIASSON, L.O.M. 2008. Atividade sazonal e uso do ambiente por anfíbios da Mata Atlântica no alto da Serra do Mar. Tese de doutorado, Instituto de Biociências da Universidadade Estadual Paulista "Júlio de Mesquita Filho", Rio Claro.

GOTELLI, N.J \& COLWELL, R.K. 2001. Quantifying biodiversity: procedures and pitfalls in the measurement and comparison of species richness. Ecol. Lett. 4: 379-391.

HADDAD, C.F.B., TOLEDO, L.F., PRADO, C.P.A., LOEBMANN, D., GASPARINI, J.L. \& SAZIMA, I. 2013. Guide to the amphibians of the Atlantic Forest: diversity and biology. Anolis Books, São Paulo.

HORTAL, J., BORGES, P.A.V. \& GASPARE, C. 2006. Evaluating the performance of species richness estimators: sensitivity to sample grain size. J. Anim. Ecol. 75: $274-287$

ICMBio (Instituto Chico Mendes de Conservação da Biodiversidade) 2015. Brazi Red Book of Threatened Species of Fauna. Ministério do Meio Ambiente. Available online at: http://www.icmbio.gov.br/portal/images/stories/comunicacao/ publicacoes/publicacoes-diversas/dcom_sumario_executivo_livro_vermelho_da fauna_brasileira_ameacada_de_extincao_2016.pdf(last access in 21/04/2017).

IUCN. 2016. The IUCN Red List of Threatened Species (Version 2015-4). Available online at: http://www.iucnredlist.org (last access in 21/04/2017).

KINDT, R. \& COE, R. 2005. Tree diversity analysis. A manual and software for common statistical methods for ecological and biodiversity studies. World Agroforestry Centre (ICRAF), Nairobi. ISBN 92-9059-179-X.

LOYOLA, R.D., LEMES, P., BRUM, F.T., PROVETE, D.B. \& DUARTE, D.S. 2014. Clade-specific consequences of climate change to amphibians in Atlantic Forest protected areas. Ecography 37: 65-72.

MAGURRAN, A.E. 2004. Measuring biological diversity. Blackwell Publishing Company, Oxford.

MALAGOLI, L.R. 2008. Anfíbios do município de São Paulo: histórico, conhecimento atual e desafios para a conservação. In Além do concreto: contribuições para a proteção da biodiversidade paulistana (L.R. Malagoli, F.B. Bajesteiro \& M Whately, eds). Instituto Socioambiental, São Paulo, p.204-231.

MALAGOLI, L.R. 2013. Diversidade e distribuição dos anfíbios anuros do núcleo Curucutu, Parque Estadual da Serra do Mar, SP. Dissertação de mestrado, Instituto de Biociências, Universidade Estadual Paulista, Rio Claro.

MITTERMEIER, R.A., GIL, P.R., HOFFMAN, M., PILGRIM, J., BROOKS, T., MITTERMEIER, C.G., LAMOREUX, J. \& FONSECA, G.A.B. 2004. Hotspots revisited: earth's biologically richest and most endangered terrestrial ecoregions. CEMEX \& Agrupacion Sierra Madre, Cidade do México.

OKSANEN, J., BLANCHET, F.G., KINDT, R., LEGENDRE, P., MINCHIN, P.R., O'HARA, R.B., SIMPSON, G.L., SOLYMOS, P., STEVENS, M.H.H. \& WAGNER, H. 2016. vegan: Community Ecology Package. R package version 2.3-3. https://CRAN.R-project.org/package=vegan (last access in 21/04/2017).

R CORE TEAM 2015. R: A language and environment for statistical computing. $R$ Foundation for Statistical Computing, Vienna, Austria. http://www.R-project. org. (last access in 30/09/2016). 
RIBEIRO, M.C., METZGER, J.P., MARTENSER, A.C., PONZONI, F.J. \& HIROTA, M.M. 2009. The Brazilian Atlantic Forest: how much is left, and how is the remaining forest distributed? Implications for conservation. Biol. Conserv. 142: 1141-1153.

SCOTT JR., N.J., \& WOODWARD, B.D. 1994. Standard techniques for inventory and monitoring: Surveys at Breeding Sites. In Measuring and monitoring biological diversity: standard methods for amphibians (W.R. Heyer, M.A. Donnelly, R.W. McDiarmid, L.C. Hayek \& M.S Foster, eds). Smithsonian Institution Press, Washington, p.118-125.
WALTHER, B.A. \& MOORE, J.L. 2005. The concepts of bias, precision and accuracy, and their use in testing the performance of species richness estimators, with a literature review of estimator performance. Ecography 28: 815-829.

Received: 19/10/2016

Revised: 18/05/2017

Accepted: 17/06/2017

Published online: 24/07/2017 